\title{
Evaluation of a Low Cost Prosthetic Hand Controlled by Surface EMG Sensors and Vibrotactile Feedback
}

\author{
Victor Argueta-Diaz \\ Assistant Professor, Physics Department \\ Alma College \\ Alma, MI 48801 \\ arguetav@alma.edu \\ Durham Basso \\ Senior, Physics Department \\ Alma College \\ Alma, MI 48801 \\ basso1dc@alma.edu
}

\author{
Cameron J. Spitzfaden \\ Graduate Student, Physics Department, Randall Lab \\ University of Michigan \\ Ann Arbor, MI 48109 \\ cspitz@umich.edu \\ Hope L. Ayers \\ Senior, Physics Department \\ Alma College \\ Alma, MI 48801 \\ ayers1hl@alma.edu
}

\begin{abstract}
This study proposes a prosthetic hand with a simple control algorithm and offthe-shelf electronics. We designed this hand to be used and repaired in underdeveloped regions. A differential sEMG signal is obtained from the flexor digitorum superficialis and extensor digitorum muscles to control the position of the hand's grasp. Three different hand control schemes (visual, vibrotactile and visual plus vibrotactile) were compared and tested in ten able-bodied individuals. We observed a better performance of the visual and vibrotactile control overall, but the vibrotactile feedback increased performance after several interactions. In subjective data evaluations vibrotactile and visual feedback had the highest scores in light and medium pressures, while visual-only feedback type had the highest average score for hard pressure.
\end{abstract}

Index Terms-Biomedical Electronics, Electromyography, Sensors, Training data, Prosthetics.

\section{INTRODUCTION}

In 2011 the World Health Organization and the World Bank published the World Report on Disability. In this report the WHO estimates that over 750 million people worldwide experience some kind of physical disability. Of those 750 million people, around $83 \%$ live in underdeveloped countries $^{1}$. Many of the state-of-the-art prosthetic devices cost thousands of dollars and require constant maintenance and expensive repair parts. There is a need, therefore, to develop low-cost and efficient prosthetic limbs for underdeveloped regions. Projects like "Jaipur Foot" have proven the relevance and need for low-cost prosthetics by providing millions of prostheses since 1975. Other organizations like "Enabling the Future" and "The Open Bionics Project" (previously the OpenHand Project) are solving this problem with the use of modern electronics and fastprototyping manufacturing.

With prosthetics users, judging the grip strength of their prosthetic is difficult. Prosthetics 
create a separation between the user and the object the user is manipulating, which can cause the patient to use an improper amount of force on the object they are manipulating. This leads to the object slipping from the person's grasp, or breaking a more fragile object. Since the main feedback system prosthetic users utilize to control their grasp is visual feedback it prevents the patient to manipulate object without a visual contact.

We can identify different kinds of feedback systems, from direct neural feedback where doctors implant electrodes into the nerve stumps of the amputated $\operatorname{limb}^{23}$, electrotactile where stimulating electrodes are placed on top of the skin, and high frequency pulses stimulate the nerves between the electrodes ${ }^{4,5,6}$, to vibrotactile feedback where haptic motors are set up so they increase the intensity of their vibration as the pressure sensed in the prosthetic increases $7,8,9,10,11,12$.

Past studies have attempted to establish the effect that vibrotactile feedback has on control of a prosthetic. Cipriani et al. in their 2008 study "On the Shared Control of an EMG-Controlled Prosthetic Hand: Analysis of User-Prosthesis Interaction", analyzed the user-prosthesis interaction, including vibrotactile feedback. They only collected visual with vibrotactile feedback data, and they realized the vibrotactile system was not being exploited because the subjects were relying on visual feedback ${ }^{7}$. They said, though, that the subjective opinion was that vibrotactile feedback was important. Cipriani's next study, "A Miniature Vibrotactile Sensory Substitution Device for Multi-fingered Hand Prosthetics" focused on the optimal placement of the haptic motor and whether humans could accurately discriminate the difference between varied amplitudes and frequencies. The researchers found that subjects were able to discriminate three different sites with an accuracy of $93 \%$ and six different stimulation patterns with an accuracy of $78 \%{ }^{8}$. In 2002, Carrozza et al. published on the fabrication of a functional prosthetic hand ${ }^{12}$ manufactured from ABS plastic using fused deposition modeling. The hand featured two identical fingers and an opposed thumb (each finger housed two DC-powered linear actuator). Their paper also discussed preliminary experiments with a peripheral nervous system interface, which involved surgical implants. In 2014, Raspopovic et al. performed a study on prosthetic hands controlled by sEMG ${ }^{11}$. They experimented with feedback from the hand to the patient to augment the sEMG control.

In this paper we present a prosthetic hand controlled with a simple algorithm and off-the-shelf electronics - not only to reduce costs, but also to allow people with limited resources and little technical background to be able to repair and replace parts of the prosthetic hand. Our hand was manufactured on a 3D printer using ABS plastic. It consists of two fingers and an opposed thumb. Each digits is controlled by a servo-motor and have a pressure sensor on its tip. The pressure sensor provides a vibrotactile feedback using haptic motors. We are focusing on vibrotactile feedback for its low-cost and non-invasive nature. Two pairs of sEMG sensors were placed on the flexor digitorum superficialis and extensor digitorum muscles. Once the sEMG signal was acquired, we controlled the position of the hand by differentiating the electric signal from each pair of electrodes and performing a simple sample-data replacement algorithm that allowed to rotate the shaft of the servo motor by a specific amount. We tested our device by recruiting ten able-bodied individuals to perform three types of grasp (light, medium and high pressure) under three different types of feedback: visual (control group), vibrotactile and vibrotactile-visual. In this paper we present the mechanical design, the testing with ten able-bodied participants and the different feedback styles as well as an analysis of the results and a summary. 


\section{Mechanics}

\section{HAND DESIGN}

The hand features two articulated fingers and an opposed thumb. Each finger comprises three segments (the thumb, two), and the segments are articulated using aluminum screw posts. Each finger is opened by elastic bands and actuated by a nylon cable. This allows each finger to be moved by a single actuator, while still conforming to the object being grasped. The palm of the hand houses three DC-powered micro servos. The servos use pulleys to actuate the nylon cables. This prototype, shown in Figure 1, was fabricated in ABS plastic with a 3D printer, but the hand could be manufactured in other materials more available in the region where the patient lives. For example, we have fabricated a hand, with similar functionality, using wood (although that hand is not presented in this paper).

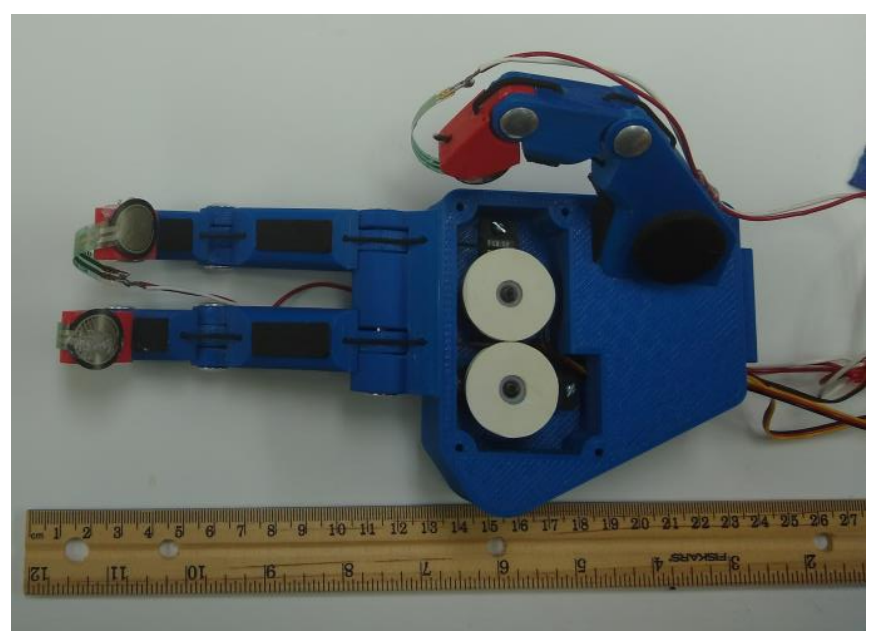

FIGURE 1

MECHANICAL PROTOTYPE

\section{Myoelectric Control}

sEMG electrodes were placed on the forearms of able-bodied subjects to determine the locations of signals useful to controlling the hand. Fairly reliable signals were found by targeting the flexor digitorum superficialis and extensor digitorum muscles (shown in figure 2), which flex and extend the fingers, respectively. These raw sEMG signals are registered through an integrated circuit, which amplifies and rectifies the signal, then calculates its time integral. The time integral of an sEMG signal is proportional to the force of muscular flexion. A microcontroller (we used an Arduino Uno for this prototype) receives the rectified and integrated sEMG signals, via analog inputs, for further processing. 


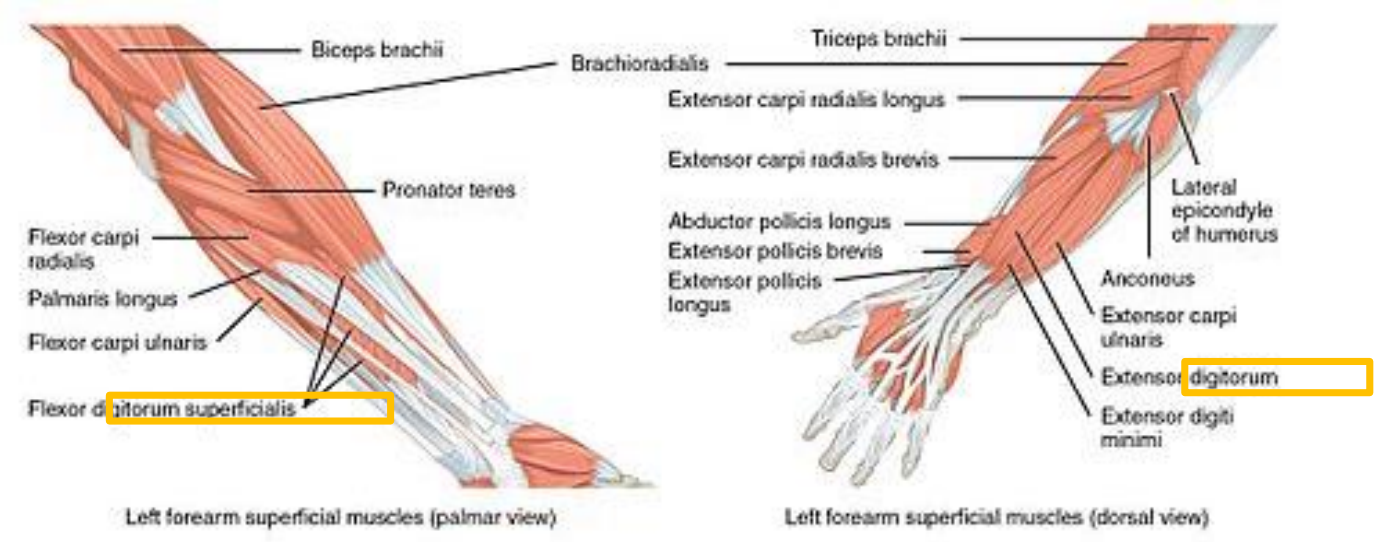

FIGURE 2

SEMG SENSORS ARE PLACED IN THE FLEXOR DIGITORUM SUPERFICIALIS AND THE EXTENSOR DIGITORUM. IMAGE VERSION 8.25 FROM THE TEXTBOOK OPENSTAX ANATOMY AND PHYSIOLOGY PUBLISHED MAY 18, 2016

We filter our sEMG signal using a a modified moving average algorithm. First we populate a 50-element array and every millisecond a new sample replaces the oldest sample in the array. As the new sample arrives we do an extreme-value-eliminating filter to remove spikes from our sample, then the average at that time can be calculated for all of the data samples using the following expression:

$$
M A_{n+1}=\frac{x_{n+1}+n \cdot M A_{n}}{n+1} \quad \text { Eq. } 1
$$

Where $M A$ is the moving average, $n$ is the number of data samples and $x_{n+1}$ is the new data sample. We do this process on each electrode targeting the extensor digitorum muscle and the flexor digitorum superficialis. The difference between the flexor and extensor signals is then calculated. The sign and magnitude of this difference determines the direction and distance of desired hand motion. If the difference remains below a threshold, the microcontroller maintains the servos at their current position. This prevents small fluctuations of the signal from perturbing the hand position, while allowing the fingers to be moved at a variety of speeds, and to arbitrary positions. Figure 3 shows the hand moving in steps, holding constant position between segments of motion. We can see in Figure 3 that when the Flexor signal is greater than the extensor (when compared to a threshold), the hand position changes, and that position can be held until the threshold is exceeded once again). A similar procedure is used to close the hand. The threshold is calculated during a calibration stage, as $25 \%$ of the signal obtain during maximum extension/flexion. 


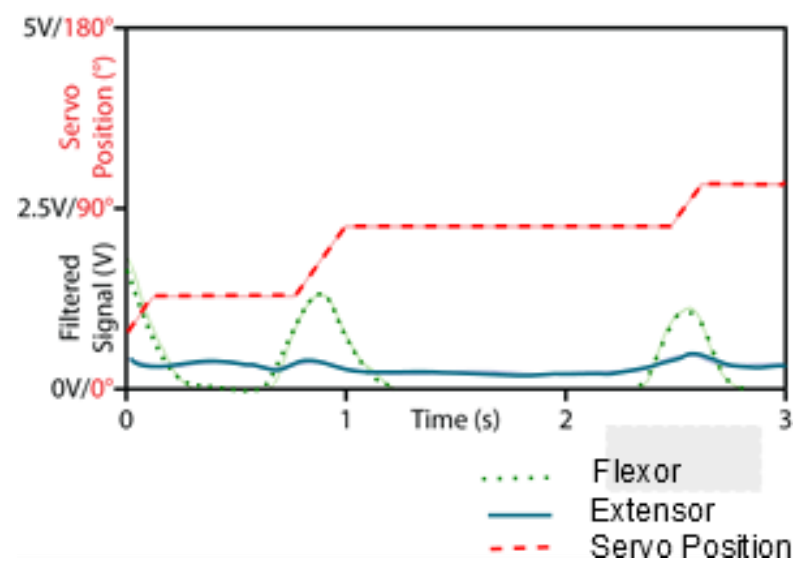

FIGURE 3

DUAL ELECTRODE CONTROL (STEPWISE MOTION OF THE HAND)

Figure 4 shows the hand signal going through the full range of motion, where 0-degrees corresponds to a fully flexed hand and 180-degrees corresponds to a fully extended hand.

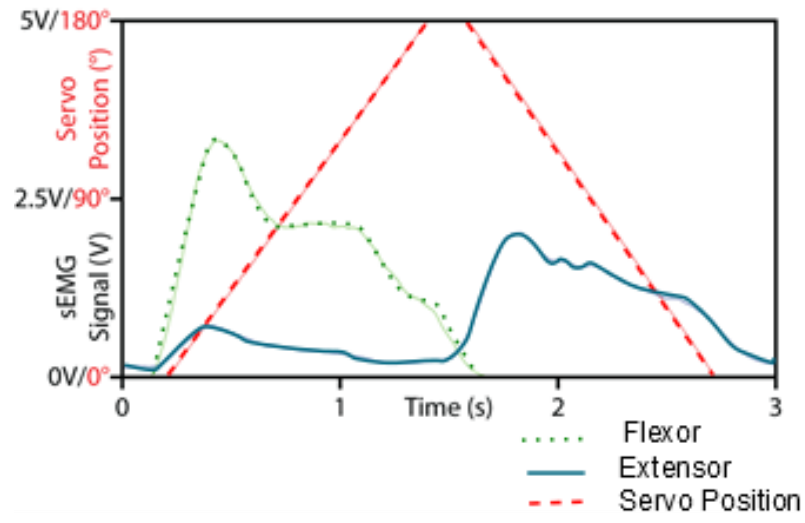

FIGURE 4

DUAL ELECTRODE CONTROL. FULL RANGE OF MOTION

\section{EXPERIMENTATION}

Ten volunteers were recruited between 18 and 38 years old. This experiment had no requirements as to age, race, gender or health. The subjects of this research were instructed that they could withdraw at any time, for any reason, without repercussion.

This study took place with the subject seated comfortably. Three electrodes were attached to the subject's dominant arm forearm along the flexor digitorum superficialis, a positive electrode, a negative and a ground. The subject's extensor digitorum muscles were also be monitored with a positive, negative and ground electrode ${ }^{10}$. The haptic motor was placed in the crook of the elbow. 
The subject was then directed through the calibration of the prosthetic. The subject started by keeping their arm relaxed on a table for three calibration sessions of 15 seconds. This gives the software controlling the prosthetic a baseline to signal "stop movement." The subject then maximally flexed his/her hand and forearm for three sessions of 15 seconds. This provided a value for the amount of muscle flexion needed to cause flexion in the prosthetic hand. Finally, the subject calibrated the extension of the prosthetic by maximally extending for 15 seconds. Once calibration was complete we allowed for 10 minutes to practice. This practice not only served the purpose of familiarizing the subject with using the prosthetic, but also began to pair the visual feedback to the vibrotactile feedback. After 10 minutes were over, he experimenter then started the subject on the vibrotactile-visual feedback trial. For each feedback type 15 trials were done, five where the subject attempted to exert a light pressure with the prosthetic, five for a medium/average pressure, and five with a hard pressure. The order of trial completion was randomized to ensure the subject did not get complacent and to minimize the effect of learning. Once the vibrotactile-visual trial was completed, a mask and noise cancelling headphone was put on the subject to block the subject's view and hearing of the prosthetic (vibrotactile only trials). As with the earlier conditions, 15 trials with this feedback type were performed with five trials of each pressure. After these trials were completed, the mask and the vibrotactile motors were removed and the visual only trials were initiated.

Quantitative data was collected by using lines traced on a sheet of paper with a pen that was attached to the prosthetic's fingers. One of these traces is shown in Figure 5. The objective of the test was for the patient to move the fingers as close as possible to one of the targets (Target 1 is for low pressure, Target 2 is for medium pressure and Target 3 is for high pressure). As they went through the trials, the prosthetic left a trail of ink that showed where the prosthetic hand traveled throughout the movements. As the fingers flexed, a set of springs increased the stress on the pressure sensors. Thus, the tighter the flex position of the hand, the higher the tension on the springs and thus a higher vibration on the haptic motor. The target lines were stenciled onto each sheet to make sure they were the same distance apart between each trial. The data recorded was the distance from the end of the prosthetics movement to the desired mark. 


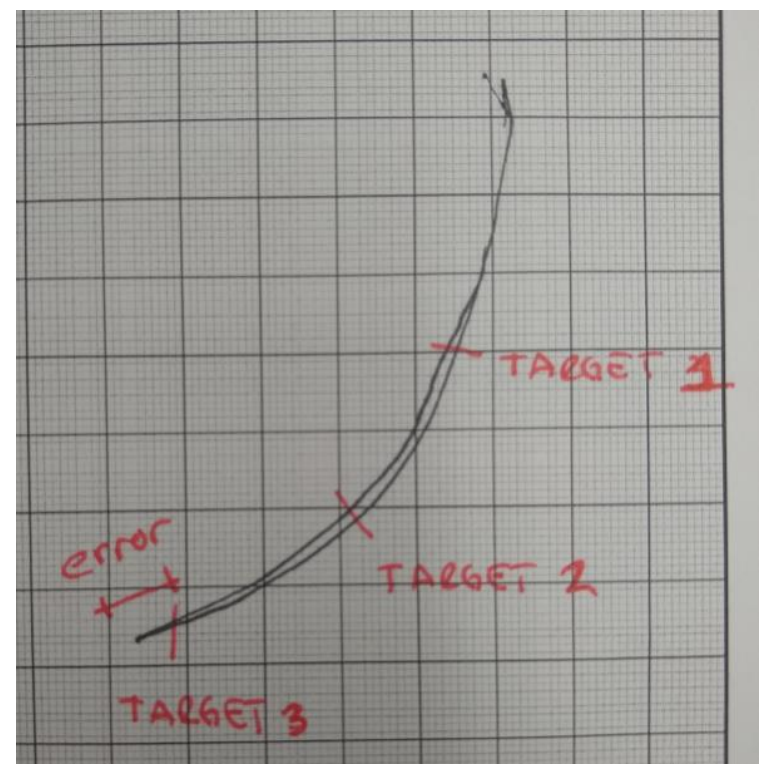

FIGURE 5

TRACE DONE BY THE PROSTHETHIC HAND WITH AN ATTACHED PEN. THE PATIENT ATTEMPTS TO MOVE THE HAND AS CLOSE AS POSSIBLE TO A TARGET (TARGET 3 IN THIS CASE). DATA COLLECTED IS THE DISTANCE FROM THE TARGET TO WHERE THE TRACE ENDS.

Upon completion of these trials the electrodes were removed from the subject and he or she was given a post-experiment survey to complete. This survey allowed the subject to subjectively score how well they thought they controlled the prosthetic in each of the feedback and pressure conditions. They also reported how comfortable they thought they were using each feedback type on a scale of 1 to 10 , one being the worst and 10 being the best.

\section{RESULTS}

\section{Quantitative Results}

In total we obtain 150 different data points for our analysis. We compare the vibrotactile feedback and the visual plus vibrotactile feedback against the visual only feedback (control group). The average distance to the target, for five different attempts, is shown in Figures 6(a-c). Each graph represents a specific kind of pressure. We can observe that the shortest distance to the target was achieved by the vibrotactile-visual feedback, while the largest distance to the target was achieved, for all three grasps, by the vibrotactile feedback. There is, however, a decreasing trend line in the vibrotactile feedback with each attempt, this is particular evident in figure $6 \mathrm{a}$ and $6 \mathrm{~b}$. In future work the authors would like to explore if a learning mechanism will make this feedback more effective. 

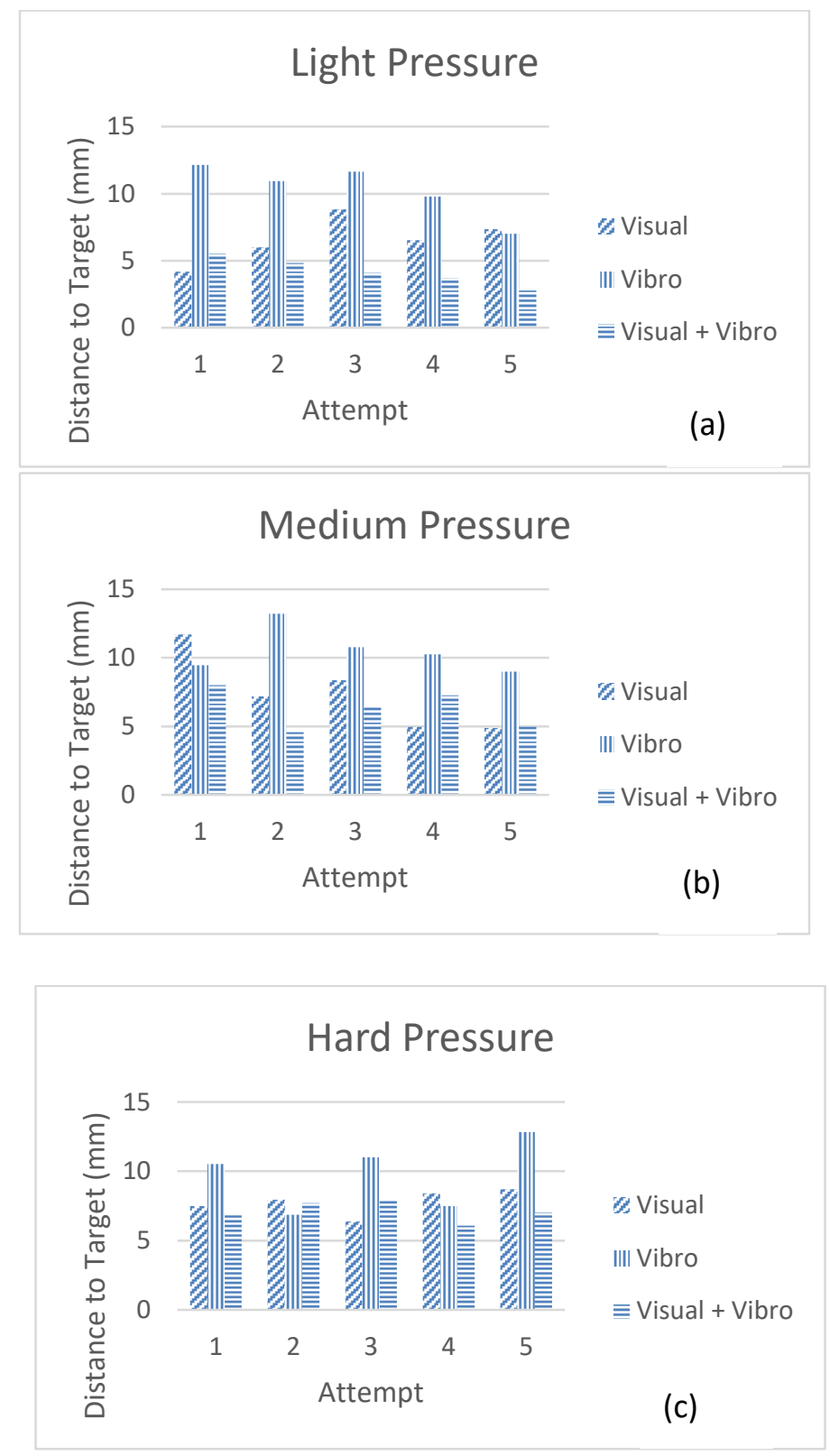

\section{FIGURE 6 \\ AVERAGE DISTANCE TO THE TARGET ON EACH GRASP FOR THREE DIFFERENT FEEDBACK SCHEMES}

Table 1 shows the average distances of each feedback system for each type of grasp. When comparing the data from the visual feedback versus the vibrotactile-visual feedback we observe an improvement in the distance to the target. The calculated p-value for both kinds of feedback against the control group was below a 0.05 significant level $(0.04$ for vibrotactile and 0.032 for vibrotactile-visual feedback for a medium grasp). Even though, further studies with a larger sample are required to guarantee a statistical significance, these results are encouraging that our proposed system has similar behavior as what has been reported previously. 


\section{TABLE I}

MEASURED AVERAGE DisTANCE-TO-THE-TARGET FOR EACH GRASP AND FEEDBACK TYPES

\begin{tabular}{|l|l|l|l|}
\hline Feedback / Grasp & Light $(\mathrm{mm})$ & Medium $(\mathrm{mm})$ & Hard $(\mathrm{mm})$ \\
\hline Visual (control group) & $7.26 \pm 0.92$ & $8.86 \pm 1.2$ & $7.5 \pm 1.34$ \\
\hline Vibrotactile & $12.5 \pm 1.3$ & $11.77 \pm 2.1$ & $9.2 \pm 1.5$ \\
\hline Visual + Vibrotactile & $5.43 \pm 0.87$ & $7.24 \pm 1.67$ & $6.22 \pm 0.87$ \\
\hline
\end{tabular}

\section{Subjective Results}

The survey results are shown in figure 7. We can see that subjects were more confident and thus felt more comfortable with each of the hard pressures (with an average of 8.2). Vibrotactile and visual had the highest scores in the light and medium pressures, but visual only feedback type had the highest average score for the hard pressure. Understandably, vibrotactile-only had the worst confidence scores for all of the pressures, but had an unexpectedly high hard pressure score. This corresponds with the quantitative data, which shows that there was an increase in the accuracy of the hard pressure of the vibrotactile only tests.

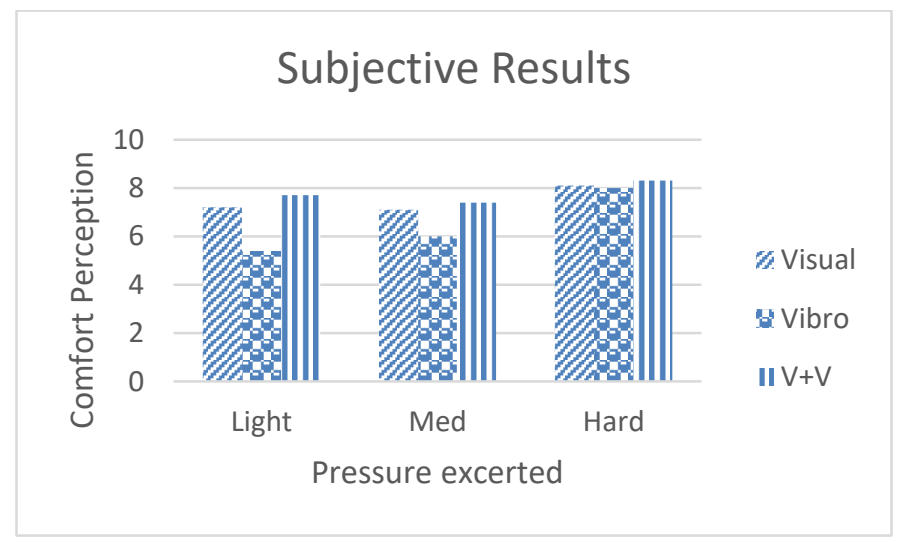

FIGURE 7

SUBJECTIVE DATA RESPONSES FOR EACH GRASP AND FEEDBACK SCHEME

\section{SUMMARY}

We have presented a prototype of a prosthetic hand fabricated in ABS plastic by a 3D printed and controlled by a microcontroller and sEMG sensors. The hand provides the user with pressure feedback by means of a pressure sensor on the finger tips and a haptic motor. The position of the hand is obtained by differentiating an sEMG signal of the flexor digitorum superficialis and the extensor digitorum. $\mathrm{W}$ also present testing of our prototype using ten able-bodied volunteers. We compare three different kind of feedback schemes: only visual, only vibrotactile, and visual plus 
vibrotactile. We measured a small improvement on the hand control using a vibrotactile-visual feedback over an only visual feedback. The improvement whether small is encouraging that our inexpensive prosthetic hand with a simple control algorithm can also be user friendly.

We also see an interesting trend on the vibrotactile-only feedback, in future work we would like to run an experiment that monitors neuron receptor desensitization over a two-week span to see if repeated teaching and exposure to the vibration frequencies would improve accuracy.

\section{REFERENCES}

${ }^{1}$ J World Health Organization. (2011). The World Bank. World report on disability. 2011. WHO Library Cataloguing-in-Publication Data. http://www.who.int/disabilities/world_report/2011/report.pdf

2 Dhillon, Gurpreet Singh, and Kenneth W. Horch. "Direct neural sensory feedback and control of a prosthetic arm." IEEE Transactions On Neural Systems and Rehabilitation Engineering 13.4 (2005): 468472.

${ }^{3}$ Kuiken, T. A., Li, G., Lock, B. A., Lipschutz, R. D., Miller, L. A., Stubblefield, K. A., \& Englehart, K. B. (2009). Targeted muscle reinnervation for real-time myoelectric control of multifunction artificial arms. Jama, 301(6), 619-628.

4 Antfolk, C., D’Alonzo, M., Rosén, B., Lundborg, G., Sebelius, F., \& Cipriani, C. (2013). Sensory feedback in upper limb prosthetics. Expert Review of Medical Devices, 10(1), 45-54.

${ }^{5}$ Szeto, Andrew YJ, and Frank A. Saunders. "Electrocutaneous stimulation for sensory communication in rehabilitation engineering." IEEE Transactions on Biomedical Engineering 4 (1982): 300-308

6 Jorgovanovic, N., Dosen, S., Djozic, D. J., Krajoski, G., \& Farina, D. (2014). Virtual grasping: Closedloop force control using electrotactile feedback. Computational and Mathematical Methods in Medicine, 2014, 120357. doi:http://dx.doi.org/10.1155/2014/120357

${ }^{7}$ Cipriani C, Zaccone F, Micera S, Carrozza MC. (2008) On the Shared Control of an EMG-Controlled Prosthetic Hand: Analysis of User-Prosthesis Interaction. IEEE Transactions on Robotics February, 170184

${ }^{8}$ Cipriani, C., D'Alonzo, M., \& Carrozza, M. C. (2012). A miniature vibrotactile sensory substitution device for multifingered hand prosthetics. IEEE Transactions on Bio-Medical Engineering, 59(2), 400408. doi:http://dx.doi.org/10.1109/TBME.2011.2173342

${ }^{9}$ Stepp, Cara E., and Yoky Matsuoka. "Relative to direct haptic feedback, remote vibrotactile feedback improves but slows object manipulation." In Engineering in Medicine and Biology Society (EMBC), 2010 Annual International Conference of the IEEE, pp. 2089-2092. IEEE, 2010.

${ }^{10}$ Kyberd, P. J., Mustapha, N., Carnegie, F., \& Chappell, P. H. (1993). A clinical experience with a hierarchically controlled myoelectric hand prosthesis with vibro-tactile feedback. Prosthetics and Orthotics International, 17(1), 56-64.

${ }^{11}$ Raspopovic, S., Capogrosso, M., Petrini, F. M., Bonizzato, M., Rigosa, J., Di Pino, G., ... \& Granata, G. (2014). Restoring natural sensory feedback in real-time bidirectional hand prostheses. Science Translational Medicine, 6(222), 222ra19-222ra19.

${ }^{12}$ Carrozza, M. C., Massa, B., Micera, S., Lazzarini, R., Zecca, M., \& Dario, P. (2002). The development of a novel prosthetic hand-ongoing research and preliminary results. IEEE/ASME Transactions on Mechatronics, 7(2), 108-114. 